\title{
Les agonistes de la LHRH en gynécologie
}

\section{André Lemay Professeur}

Rodolphe Maheux Associé de recherche

\section{Nacia Faure}

Professeur
Remerciements

Les auteurs tiennent à remercier le Dr Chantal Guilloteau des échanges France-Québec pour sa participation à la rédaction et Louise Mercier pour la préparation du manuscrit.

\section{ADRESSE}

A. Lemay, R. Maheux, N. Faure : département de gynécologie et obstétrique de l'université Laval ct département de recherche et centre d'endocrinologie de la reproduction ct d'infertilité de l'hôpital Saint-François-d'Assise, Québec,

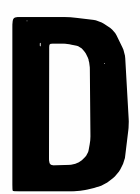
epuis la purification en I97 I [I] du décapeptide LHRH (luteinizing hormone releasing hormone), hormone hypothalamique responsable de la sécrétion hypophysaire de LH (luteinizing hormone) et de FSH (follicle-stimulating hormone), un très grand nombre de dérivés à action agoniste puis antagoniste ont été synthétisés en laboratoire. Le développement de dérivés agonistes de la LHRH a été rapide. Une simple substitution sur le $6^{\text {e }}$ acide aminé, avec ou sans remplacement du $10^{\mathrm{e}}$ acide aminé par un groupement éthylamide, bloque la dégradation enzymatique, ce qui accroît et prolonge l'activité biologique.

Les progrès dans la synthèse de dérivés antagonistes ont été plus tardifs car de nombreuses substitutions doivent être faites sur la molécule de LHRH. Les essais cliniques sur les antagonistes nouvellement synthétisés les plus prometteurs débutent et nécessitent des quantités encore importantes de produits (plus de $100 \mu \mathrm{g} / \mathrm{kg}$ ).

\section{Actions}

\section{pharmacologiques}

Une stimulation maximale de la sécrétion de gonadotrophines peut être obtenue chez l'homme par l'injection sous-cutanée ou intra- veineuse de petites quantités d'agoniste $(0, \mathrm{I}$ à $0,5 \mu \mathrm{g} / \mathrm{kg})$. La voie intra-nasale est utilisée mais l'absorption est faible; l'action sur l'hypophyse n'est que de 3 à $5 \%$ de celle obtenue par une injection sous-cutanée [2].

Étant donné qu'une stimulation maximale de la sécrétion de gonadotrophines est obtenue avec les agonistes, l'objectif initial de l'utilisation de ces dérivés de la LHRH était d'obtenir une action prolongée pouvant stimuler l'axe hypophysoovarien pour le traitement de l'anovulation, de l'aménorrhée, de l'hypogonadisme... etc. Mais les premiers essais cliniques ont eu peu de succès et des effets inhibiteurs paradoxaux sont apparus. Dans plusieurs espèces animales, divers traitements par administration de doses pharmacologiques de LHRH ou d'agonistes de la LHRH ont inhibé l'ovulation, induit une lutéolyse ou entraîné un avortement [3]. Chez l'homme, il est bien connu maintenant que la $\mathrm{LH}$ et la FSH sont sécrétées de façon pulsatile, avec une fréquence moyenne d'environ I pulsation toutes les $90 \mathrm{mi}-$ nutes. La perte de pulsatilité après administration d'agoniste de la LHRH explique les effets inhibiteurs paradoxaux. L'effet sur l'axe hypophyso-ovarien est biphasique (figure I). La stimulation initiale, de courte durée, est suivie d'une 


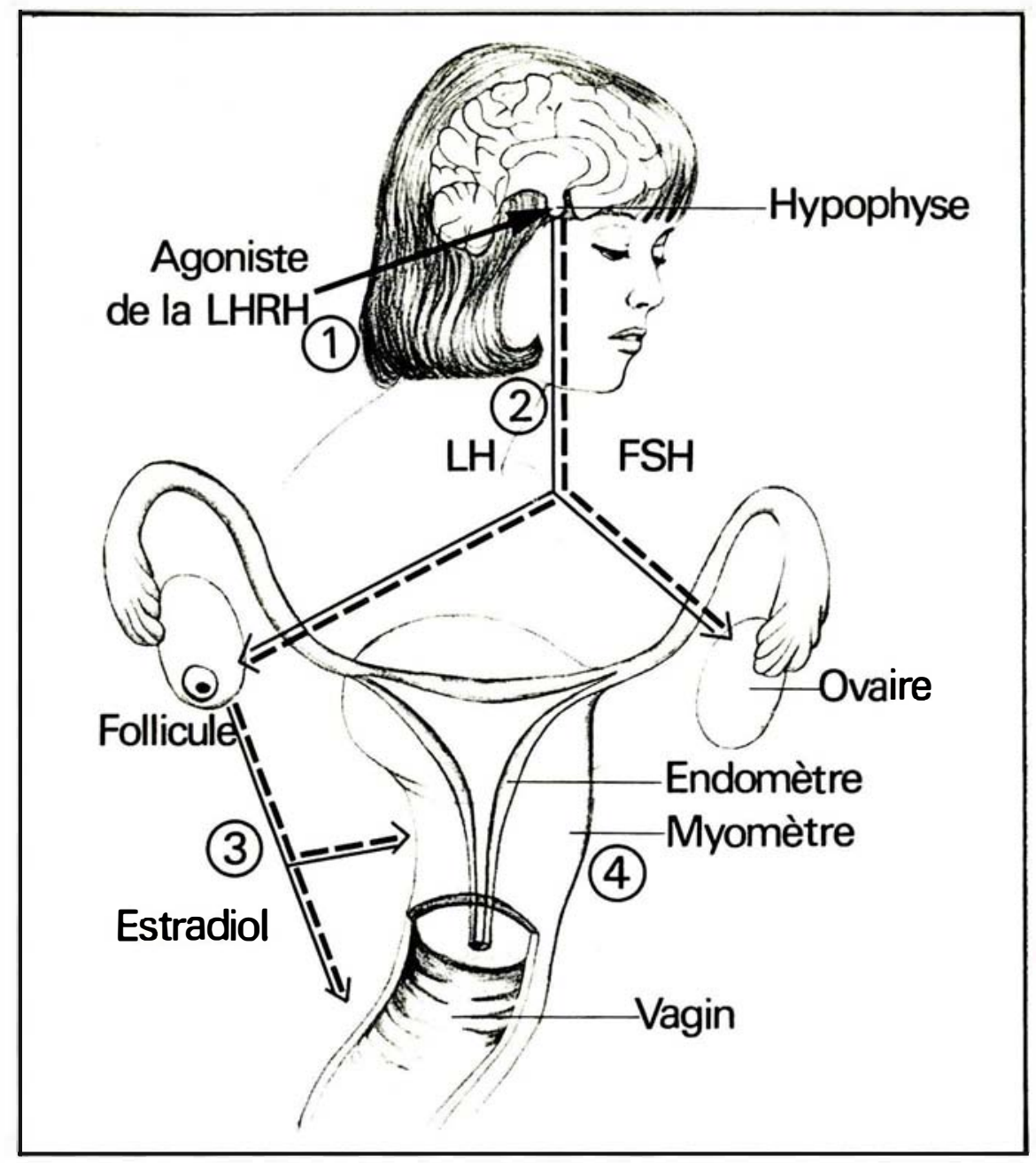

Figure 1. Schéma illustrant l'enchaînement des étapes de la réponse à I'administration d'un agoniste de la LHRH chez la femme.

(1) Administration d'agoniste de la LHRH par voie sous-cutanée ou intra-nasale. (2) Réponse hypophysaire en LH et en FSH. (3) Effets sur l'ovaire et l'estradiol. (4) Effets sur les tissus reproducteurs : myomètre, endomètre, muqueuse vaginale. stimulation initiale; - _ - inhibition secondaire.

inhibition. Celle-ci se traduit par une diminution de la concentration des hormones sexuelles et de leurs effets sur les organes cibles, principalement l'utérus et le vagin. La réponse au traitement par les agonistes de la LHRH dépend de la dose et de la fréquence d'administration.

\section{Administration unique}

L'administration de doses croissantes de [D-Ser(TBU ${ }^{6}$-Des-Gly$\mathrm{NS}_{2}^{10}$ ] LHRH éthylamide (Busereline) cause une libération prolongée de gonadotrophines remplaçant les pulsations normales par une et le processus d'ovulation [4]. Il a été démontré chez l'animal que l'élévation supra-physiologique de la concentration de gonadotrophines entraîne une désensibilisation des récepteurs de ces hormones dans les ovaires [5]. Ce phénomène n'a pas encore été étudié chez l'homme. Des changements aigus des stéroïdes intra-ovariens pourraient également modifier les processus physiologiques de l'ovaire. Une action directe des agonistes de la LHRH à ce niveau a été mise en évidence chez l'animal [6]. Si l'existence de récepteurs de la LHRH a été confirmée dans l'hypophyse, leur présence n'a pas été prouvée dans le tissu ovarien humain [7]. Par ailleurs, dans les cellules de granulosa mises en culture, des doses croissantes d'agonistes de la LHRH ont pu inhiber la sécrétion basale de progestérone [8] mais n'ont pas pu empêcher l'effet stimulateur de la FSH [9].

\section{Administration répétitive}

L'hypophyse humaine est réfractaire à une seconde administration intra-nasale d'agoniste de la LHRH dix heures après un premier traitement [Io]. L'administration d'une dose plus élevée ou à une fréquence plus grande conduit à une perte plus rapide de la réponse hypophysaire [I I ]. La réponse à l'administration répétitive d'agoniste de la LHRH est schématisée dans la figure 2 (voir page suivante).

L'injection quotidienne de $200 \mu \mathrm{g}$ par voie sous-cutanée ou l'insufflation intra-nasale de $400 \mu \mathrm{g}$ trois fois par jour commencée en début de phase folliculaire, entraine un effet biphasique [I I ]. L'élévation des gonadotrophines en début de traitement stimule un développement folliculaire variable sur une période de une à trois semaines. Les niveaux sériques de FSH s'effondrent après deux à trois jours de traitement, alors que les taux immunologiques de la LH demeurent supéricurs à ceux existant en période préthérapeutique durant quelques semaines. Après une à trois semaines, les concentrations d'estradiol diminuent et deviennent alors inférieures à celles rencontrées au début 


\section{REEFERENCES}

I. Schally A V, Kastin A J, Arimura A. FSHreleasing hormone and $\mathrm{LH}$ releasing hormone. Vitam Horm 1972; 30: 83-164.

2. Lemay A, Metha A E, Tolis G, Faure N Labrie F, Fazekas A T A. Gonadotropins and estradiol responses to single intranasal or subcutaneous administration of a luteinizing hormonereleasing hormone agonist in the carly follicular phase. Fertil Steril 1983; 39: 668-73.

3. Corbin A, Bex F J. Physiology and contraceptive eff ects of LHRH and agonistic analogs in female animals. In: Zatuchni G I, Shelton J D, Sciarra J J, eds. LHRH Peptides as Female and Male Contraceptives. Philadelphia: Harper \& Row Publishers, 1981: 68-84.

4. Lemay A, Faure N, Labrie F, Fazekas A T A Acute and intermittent LHRH agonist dosing as post-coital and antiovulatory contraceptive approaches. In: Runnebaun, ed. Future Aspects in Contraception. MTP Press Ltd (sous presse).

5. Kledzik G S, Cusan L, Auclair C, Kelly P A, Labrie $F$. Inhibition of ovarian luteinizing hormone ( $\mathrm{LH})$ and follicle-stimulating hormone receptor levels by treatment with an $\mathrm{LH}$-releasing hormone agonist during the estrous cycle in the rat. Fertil Steril 1978; 30:348-53.

6. Hsueh A J W, Erickson G F. Extrapituitary action of gonadotropin-rcleasing hormone: direct inhibition of ovarian steroidogenesis. Science 1979; 204: 854-5.

7. Clayton R N, Huhtaniemi I T. Absence of gonadotropin-releasing hormone receptors in human gonadal tissue. Nature 1982; 229: 56-9.

8. Tureck R W, Mastroianni L, Blasco L, Strauss J F. Inhibition of human granulosa cell progesterone secretion by a gonadotropin-releasing hormone agonist. 7 Clin Endocrinol Metab 1982; 54: $1078-80$.

9. Casper R F, Erickson G F, Rebar R W, Yen S $S C$. The effect of luteinizing hormone-releasing factor and its agonist on cultured human granulosa cells. Fertil Steril 1982; 37: 406-9.

ro. Lemay A, Faure N, Labrie F, Fazekas A T A. Gonadotroph and corpus luteum responses to two successive intranasal doses of a luteinizing hormone-releasing hormone agonist at different days after the mid-cycle luteinizing hormone surge. Fertil Steril 1983; 39: 661-7.

II. Lemay A, Maheux R, Faure N, Jean C, Fazekas A T A. Reversible hypogonadism induced by 'a luteinizing hormone-releasing hormone (LHRH) agonist (buserelin) as a new therapeutic approach for endometriosis. Fertil Steril I 984; 4 I: 863-71. de la phase folliculaire.

Après un mois de traitement par injection chronique d'agoniste de la LHRH, on a pu démontrer que les pulsations de gonadotrophines étaient abolies [12]. L'activité biologique de la $\mathrm{LH}$ diminue beaucoup plus rapidement que son activité immunologique [I3]. Après arrêt des traitements, on observe un retour rapide du cycle menstruel normal [I I]. Ainsi, dans l'espèce humaine, le mécanisme principal expliquant l'effet inhibiteur para- doxal de l'administration répétitive des agonistes de la LHRH est une inhibition réversible de la sécrétion de gonadotrophines conduisant à un état similaire à celui rencontré dans l'hypogonadisme hypogonadotrophique.

La possibilité d'induire un hypogonadisme hypogonadotrophique par un agoniste de la LHRH a ouvert la voie à plusieurs possibilités de traitement des maladies gynécologiques liées à la sécrétion des hormones ovariennes.

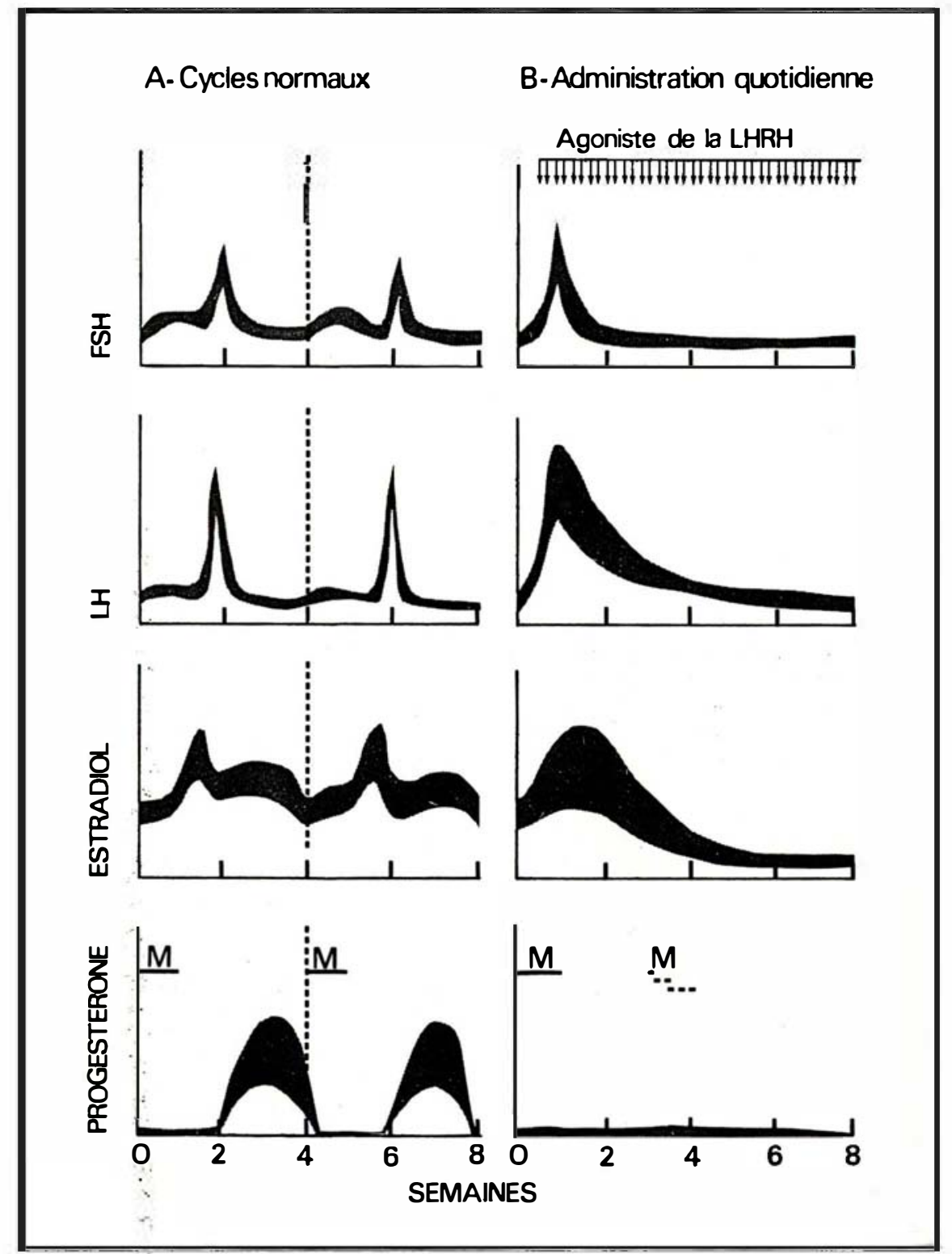

Figure 2. Schéma de l'effet de l'administration chrónique d'un agoniste de la LHRH sur l'axe hypophyso-ovarien. $M$ : menstruations. 
Les causes de l'endométriose ne sont guère connues, mais on observe que le tissu endométrial ectopique est sensible aux hormones ovariennes du cycle menstruel et cesse de se développer à la ménopause.

\section{Endométriose et léiomyome utérin}

La possibilité de supprimer de façon sélective la sécrétion ovarienne par l'administration d'agonistes de la LHRH constitue une nouvelle forme médicale du traitement de cette maladic.Les résultats obtenus dans un premier cas [14] et dans une étude pilote portant sur io patientes [I I] ont démontré qu'un traitement par un agoniste de la LHRH sur une période de six mois avait des effets bénéfiques sur la maladic. Après quelques semaines de traitement, les douleurs pelviennes s'atténuent et, à la fin du traitement, les implants de glandes endométriotiques identifiés par laparoscopie ont disparu (figure 3). A l'arrêt de la thérapeutique, on observe un retour rapide du cycle menstruel et de l'ovulation. Six mois plus tard, les symptômes d'endométriose sont toujours absents et l'examen gynécologique est satisfaisant, ce qui témoigne d'une stabilisation de la maladie. Trois cas de grossesses ont été obscrvés à la suite de ce traitement.

L'étiologie du léiomyome utérin est également inconnue. Cependant, l'expérience clinique montre qu'il est clairement estrogéno-dépendant. En effet, tout comme l'cndométriose, cette tumeur n'est pas présente avant la puberté et régresse à la ménopause. L'utilisation des agonistes de la LHRH pouvant supprimer sélectivement les estrogènes constitue également une façon élégante d'étudier la physiopathologie de cette tumeur, première cause actuelle d'hystérectomie.

Une étude pilote utilisant l'injection sous-cutanée d'agonistes de la LHRH sur une période de six mois a montré une régression rapide des léiomyomes utérins [15]. Sept des douze léiomyomes utérins observés ont présenté une régression marquée en fin de traitement, deux n'étant plus détectables à l'échographic, alors que cinq autres

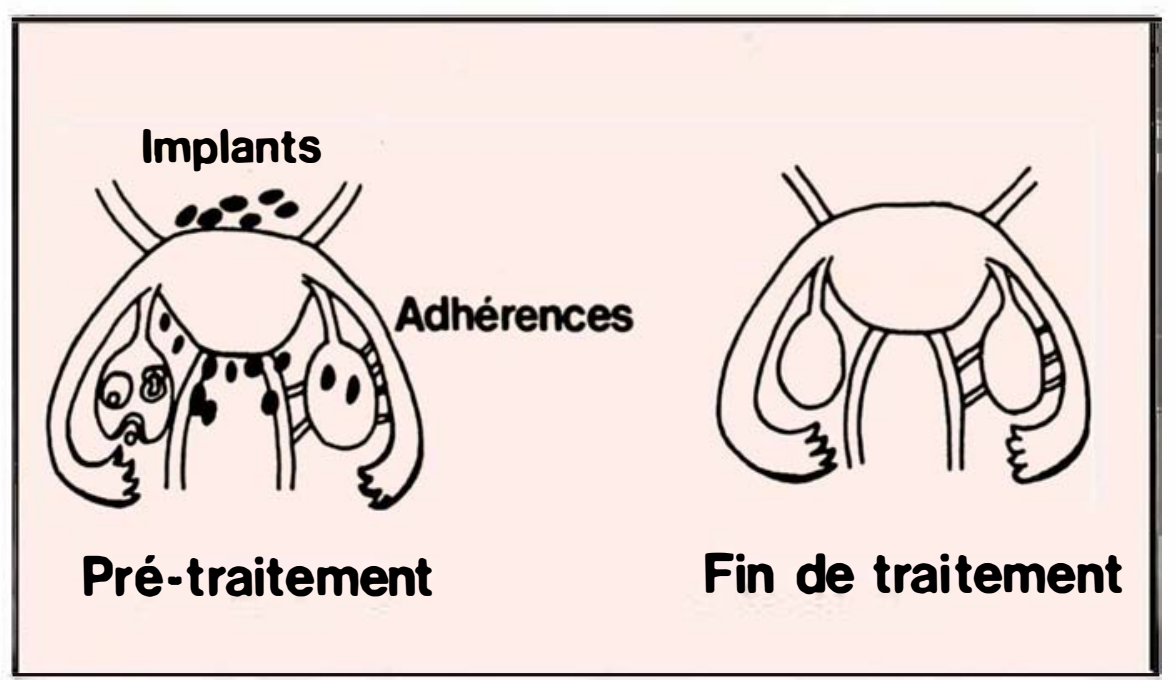

Figure 3. Schéma de la régression des implants d'endométriose à la laparoscopie après six mois de traitement par un agoniste de la LHRH.

avaient diminué d'au moins $80 \%$ (figure 4, voir page suivante). Ce traitement est particulièrement recommandé chez la femme infertile, chez la femme ayant eu des avortements spontanés et chez la jeune femme ayant un léiomyome utérin symptomatique et désirant préserver son potentiel de fertilité. Dans ce cas, il pourrait être utilisé seul ou être associé à une myomectomie après régression de la tumeur. A l'approche de la ménopause, un traitement d'entretien pourrait permettre d'éviter de pratiquer une hystérectomie.

\section{Syndrome des ovaires polykystiques}

Il est de plus en plus évident que le syndrome des ovaires polykystiques est dû à une dysfonction hypophysaire. Dans de nombreux cas, on peut mettre en évidence une hypertonie de $\mathrm{LH}$ entretenant le cercle vicieux de la lutéinisation des follicules ovariens. On a pu récemment démontrer qu'après un mois de traitement par injection souscutanée d'agoniste de la LHRH, les niveaux sériques primitivement élevés d'androstènedione et de testostérone étaient ramenés à des niveaux comparables à ceux obtenus généralement après l'ablation des ovaires [12]. Dans cette étude à court terme (un mois), la sécrétion des hormones surrénaliennes n'avait pas changé. L'emploi des agonistes de la LHRH pourrait donc être un moyen de rétablir l'équilibre hormonal et de permettre l'étude physiopathologique de cette maladie. Il sera intéressant d'évaluer la possibilité d'ovulation spontanée ou d'induction de l'ovulation après quelques semaines de traitement.

\section{Saignements utérins dysfonctionnels}

En l'absence de maladie caractérisée, il n'existe pas actuellement de traitement médical idéal pour les femmes présentant des saignements menstruels excessifs ou irréguliers. L'hormonothérapie cyclique à base de progestatifs utilisés seuls ou combinés à des estrogènes est efficace, mais elle peut avoir des effets secondaires ou être contre-indiquée. Une étude préliminaire chez quatre patientes âgées de 39 à 44 ans, présentant des ménorragies ovulatoires inexpliquées a montré une réduction spectaculaire des pertes sanguines [16]. Ces résultats ont été obtenus à l'aide d'une petite dose d'agoniste de la LHRH (200 Hg) administrée par voie intra-nasale trois fois par jour. Dans les cas de 


\section{REFERENCES}

12. Chang R J, Lauf cr L R, Mcldrum I) R, (' al. Stcroid sccretion in polycystic ovarian discasc after ovarian suppression by a long-acting gonadotropin-rclcasing hormonc agonist. $7 \mathrm{Clin}$ Endocrinol Metab 1983; 56: 897-903.

13. Mcldrum D R, Tsao Z, Monroc S E, et al. Stimulation of $\mathrm{LH}$ fragments with reduced bioactivity following GnRH agonist administration in women. 7 Clin Endocrinol Metab 1984; 58: 755-7.

14. Lemay A, Quesnel G. Potential new treatment of endometriosis: reversible inhibition of pituitary-ovarian function by chronic intranasal administration of a lutcinizing hormoncrelcasing hormonc (LH-RH) agonist. Fertil Steril 1982; 38: 376-9.

15. Mahcux R, Guillotcau C, Lemay A, Bastide A, Fazckas A T A. Regression of lciomyomata uteri following hypoestrogenism induced by repctitive lutcinizing hormonc-rclcasing hormone agonist trcatment: preliminary report. Fertil Steril 1984; 42: $644^{-6}$

16. Shaw R N, Fraser H M. Usc of a supcractive lutcinizing hormone releasing hormone (LHRH) agonist in the treatment of menorrhagia. $\mathrm{Br} F$ Obstet Gynaecol 1984; 91: 913-6.

17. Flcming $R$, Hamilton $M P R$, Barlow $D H$, Cardiner J N, Coutts J R T. Pregnancy af ter ovulation induction in a paticnt with menopausal gonadotropin levels af ter chemotherapy. Lancet 1984; i: 399.

I8. Porter R N, Smith N, Craft I L, Abdulwahid $\mathrm{N} A$, Jacobs $\mathrm{H} \mathrm{S}$. Induction of ovulation for in vitro fertilisation using buscrelin and gonadotropins. l.ancet 1984; ii: 1284-5.

19. Musc K M, Celd N S, Futterman L A, Yen $S$ S C. The premenstrual syndrome. Effects of medical ovaricctomy. $N$ Engl 7 Med 1984; 31r: 1345-9.

20. Mansficld M J, Bcardsworth D E, Loughlin J $S$, et al. Long term treatment of central precocious pubcrty with a long-acting analoguc of lutcinizing hormonc-rclcasing hormonc. $N$ Engl f Med 1983; 309: $1286-90$.

21. Klijn J G M, De Jong $F$ H. Long-tcrm treatment with the LHRH-agonist Buscrelin (HOE 766) for metastatic brcast cancer in single and combined drug regimens. In: Labric $F$, Bćlanger A, Dupont A, cds. $L H R H$ and its Analogue's. Basic and Clinical Aspects. Amstcrdam: Elscvicr Science Publishers, 1984: 396-404.

22. Lemay A, Mahcux R, Faurc N, Jcan C, Fazekas A T A Efficacy and safety of LHRH agonist treatment in 10 paticnts with endometriosis. In Labric F, Bélanger A, Dupont A, cds. $L H R H$ and its Analogues. Basic and Clinical Aspects. Amstcrdam: Elscvier Science Publishers, I984: 383-95. saignements utérins dysfonctionnels anovulatoires, il est également possible d'utiliser des agonistes de la LHRH de manière à induire une aménorrhée de courte durée, puis de réduire la posologic de manière à maintenir des taux d'estrogènes égaux à ceux de la phase folliculaire. Des saignements périodiques sont provoqués par l'administration complémentaire de progestatifs.
L'inhibition ou le contrôle des gonadotrophines par l'administration répétitive d'agonistes de la LHRH pourrait s'appliquer à plusieurs autres maladies gynécologiques. Par exemple : chez une patiente ayant des niveaux élevés de LH et FSH consécutifs à une chimiothérapie pour une maladic de Hodgkin, on a pu réduire les concentrations de gonadotrophines
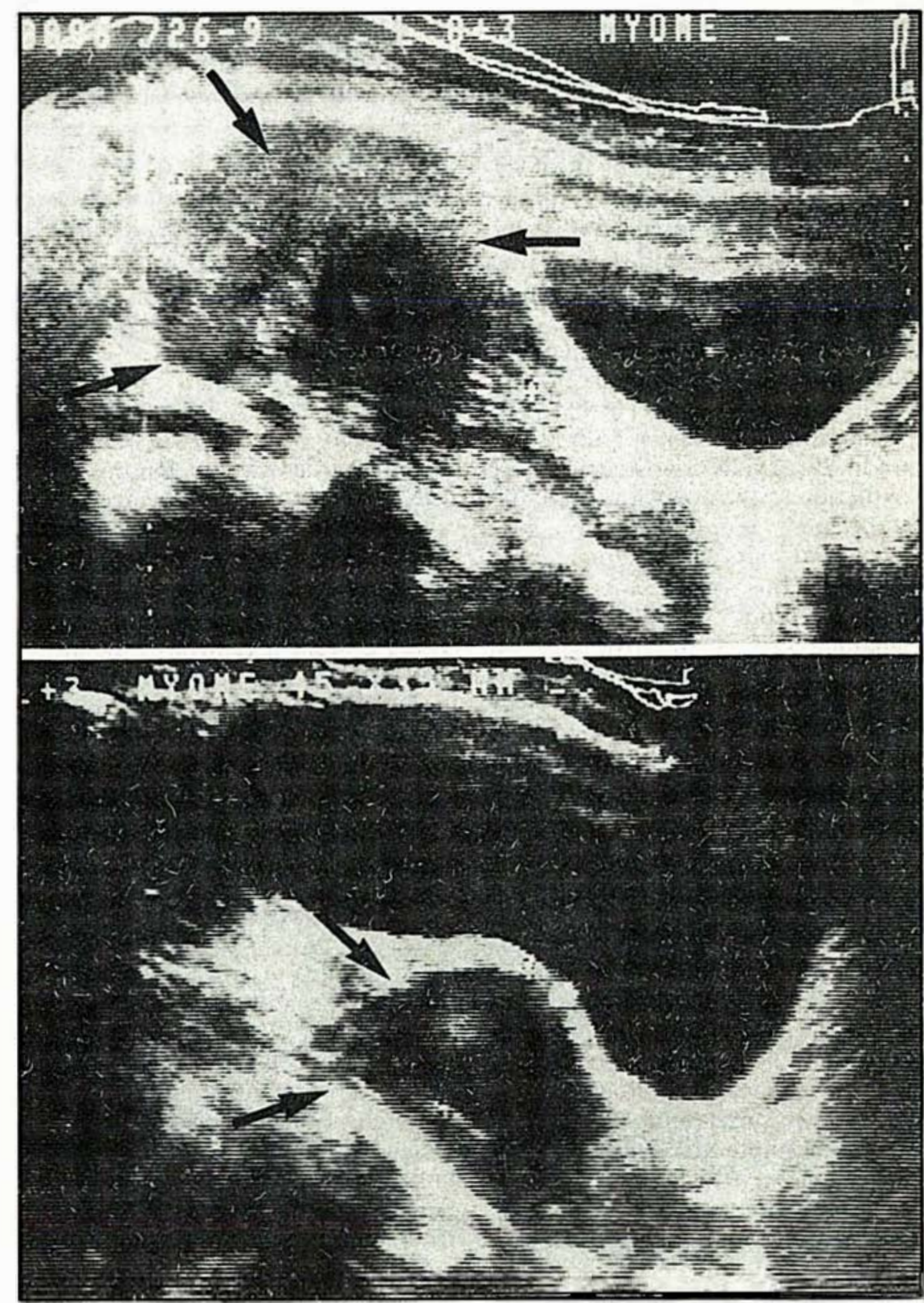

Figure 4. Echographie pelvienne montrant une diminution de $90 \%$ du volume d'un léiomyome utérin après six mois de traitement par un agoniste de la LHRH. 
circulantes et induire une ovulation (couronnée d'une grossesse normale) durant un traitement par des agonistes de la LHRH [1 7]. Ainsi, il serait possible d'utiliser un tel traitement pour abaisser le taux de gonadotrophines dans les cas "d'ovaires résistants " avec niveaux sériques élevés de $\mathrm{LH}$ et de FSH. Lors d'un traitement suppressif, il serait alors intéressant d'étudier l'administration de gonadotrophines exogènes dans le but d'induire l'ovulation chez ces femmes désirant une grossesse. Un traitement similaire de suppression temporaire de l'ovaire pourrait être utile pour la stimulation de follicules multiples en vue de la fertilisation extracorporelle [18]. D'autre part, l'inhibition de l'ovulation et des estrogènes lors de traitement par agoniste de la LHRH soulagerait les douleurs associées au syndrome pré-menstruel sévère [19]. Dans ce cas, l'addition périodique d'un progestatif pourrait être une alternative valable de traitement.

Les agonistes de la LHRH sont actuellement utilisés dans le traitement de la puberté précoce idiopathique et cette médication s'avère des plus prometteuses [20]. La possibilité de causer une véritable castration chimique est également une alternative très intéressante pour le traitement du cancer du sein hormono-dépendant chez la femme pré-ménopausée [2 I]

\section{Effets secondaires}

Les effets secondaires d'un traitement suppressif par agoniste de la LHRH sont liés à l'effet biphasique sur la sécrétion d'estrogène [ I I ]. Ils dépendent également de la dose et de la fréquence d'administration. Après la stimulation initiale de la sécrétion d'estrogènes, un saignement utérin de courte durée peut être observé pendant les premières semaines de traitement. Par la suite, la diminution de la concentration d'estrogènes est responsable de bouffées de chaleur d'intensité variable, de sécheresse vaginale et, à l'occasion, d'une baisse de la libido. Par ailleurs, il n'y a pas d'autre effet secondaire de la prise médicamenteuse et le traitement est en général très bien toléré par les patientes.
Depuis le début des études cliniques avec les agonistes de la LHRH, l'innocuité de cette nouvelle molécule a été vérifiée au cours de l'administration intensive ou modérée et régulière du traitement. Les tests de laboratoire concernant l'évaluation habituelle de la formule sanguine et des examens biochimiques du sang n'ont montré aucun effet biologique des traitements [22]. Au cours d'études à long terme, dans le cancer de la prostate en particulier, aucun changement significatif n'a été mis en évidence. Chez la femme, les seuls changements biologiques observés sont la chute des taux des hormones sexuelles et une légère diminution des niveaux sériques de prolactine.

\section{Perspectives éventuelles}

Des études multicentriques sont actuellement en cours afin de valider l'efficacité des agonistes de la LHRH dans le traitement de l'endométriose. La suppression complète des ovaires ne pourrait cependant constituer un traitement à long terme en raison des effets indésirables d'une carence estrogénique prolongée sur la masse osseuse et sur les lipoprotéines de haute densité. Chez les patientes devant recevoir un traitement d'entretien, il est probable qu'il sera possible d'établir une posologie susceptible de prévenir une recrudescence de la maladie tout en préservant une sécrétion estrogénique suffisante pour l'organisme. Le développement de nouvelles voies d'administration, implants sous-cutanés principalement, facilitera l'utilisation des agonistes de la LHRH. Pour éviter les inconvénients de la stimulation folliculaire initiale, le traitement pourrait débuter en fin de phase lutéale.

\section{Conclusion}

Les agonistes de la LHRH constitueront donc une médication de plus en plus employée pour le contrôle ou l'inhibition de la fonction ovarienne. De nombreuses possibilités d'application sont offertes en pathologie gynécologique si l'on prend soin d'éviter l'hypoestrogénisme prolongé

\section{Summary}

Depending on the dosage and frequency of administration, LHRH agonist dosing downregulates the gonadotropins, and serum estrogens can be suppressed to menopausal levels. Ovarian activity rapidly returns after cessation of treatment. This reversible state of hypogonadotrophic hypogonadism is being used for new therapeutic approaches of endometriosis, leiomyoma uteri, polycystic ovarian disease and dysfunctional uterine bleeding. LHRH agonist treatment could be used for gonadotropin-induced follicular development in ovarian resistant syndrome and in vitro fertilization, could be useful in severe premenstrual syndrome and is very promising in idiopathic early puberty. LHRH agonist chemical castration could be an alternative treatment to estrogen-dependent breast cancer in premonopausal women. Clinical side effects are related to the degree of estrogen deprivation (hot flushes, vaginal dryness...). No changes have been documented in usual biological safety parameters. Slow release delivery systems are being investigated. For long term therapy, regimens could be developed that would preserve adequate estrogen secretion.

\section{TIRES A PART}

A. Lemay : département de gynécologic et obstétrique de l'université Laval et département de recherche et centre d'endocrinologic de la reproduction et d'infertilité de l'hôpital SaintFrançois-d'Assisc, Québec, Canada. 